\title{
Anti-fibrotic Effects of Rhus javanica Linn (Anacardiaceae) Extract against Activated Hepatic Stellate Cells via Regulation of TGF-beta and Smad Signaling
}

\author{
Seung-Hoon Yoo ${ }^{1}$, Chan-Jin Yoon ${ }^{1}$, Dong-Ha Kim ${ }^{1,4}$, Mun-Jeong Yum ${ }^{1}$, Jin- \\ Seoub Kim ${ }^{1}$, Yeo-Chan Yoon ${ }^{2}$, Chi-Su Chun ${ }^{2}$, Jae-Dong Lee ${ }^{3}$, Sushruta \\ Koppula $^{5 *}$ and MinDong Song ${ }^{1,5 *}$ \\ ${ }^{1}$ Department of Applied Life Science, Graduate School of Konkuk University, Chungju, Chungbuk, ${ }^{2}$ Food One Corp, 174-45, \\ Daseseongro, Daeso-myeon, Eumseong-Gun, ${ }^{3}$ Department of Internal Medicine, School of Medicine, Konkuk University, \\ Chungju, Chungbuk, ${ }^{4}$ Asan Institute of Life Sciences, University of Ulsan College of Medicine, Poongnap-Dong, Songpa-Gu, \\ Seoul, ${ }^{5}$ Department of Biotechnology, College of Biomedical and Health Sciences, Konkuk University, Chungju, Chungbuk, \\ 380-701, South Korea
}

*For correspondence: Email: minds@kku.ac.kr, koppula@kku.ac.kr; Tel/Fax: +82-43-840-3612, +82-43-840-3609

\begin{abstract}
Purpose: To evaluate the anti-fibrotic effects of ethanol extract of Rhus javanica Linn. (Anacardiaceae) (RJE) in activated hepatic stellate cells (HSCs) as well as explore the underlying mechanisms.

Methods: The cytotoxic effect of RJE (100, 300 and $500 \mu \mathrm{g} / \mathrm{mL})$ was analyzed using 3- $[4,5-$ dimethylthiazol-2-yl]-2, 5-diphenyl tetrazolium bromide (MTT) assay in Chang liver cells. The mRNA expression of collagen type I, alpha 2 (COL1A2), transforming growth factor-beta (TGF- $\beta$ ), $\alpha$-smooth muscle actin ( $\alpha$-SMA) and platelet-derived growth factor (PDGF) were determined using reverse transcription-polymerase chain reaction (RT-PCR) in HSCs. Protein expression of collagen and Smad were measured by Western blot analysis.

Results: Treatment with RJE extract at 100, 300 and $500 \mu \mathrm{g} / \mathrm{mL}$ did not show any signs of cytotoxicity to Chang liver cells. RJE at $500 \mu \mathrm{g} / \mathrm{mL}$ concentration influenced the morphology, reduced the stretched fiber and decreased the number of viable cells in activated HSCs. The increased expressional levels of fibrosis mediators such as COL1A2, TGF- $\beta, \alpha-S M A$ were decreased by RJE (500 $\mu \mathrm{g} / \mathrm{mL})$ pre-treatment. Quantification data showed that the increased band intensity of COL1A2 (1.41 \pm 0.08$)$, TGF- $\beta$ (1.23 \pm $0.13), \alpha-S M A(1.71 \pm 0.14)$ were significantly $(p<0.05)$ reduced to $0.39 \pm 0.12,0.35 \pm 0.11$ and $0.04 \pm$ 0.08 , respectively upon RJE treatment. However, RJE did not suppress the expression of PDGF gene. Mechanistic study revealed that RJE prevented fibrosis in HSCs via regulation of TGF- $\beta$ and Smad signaling pathways.

Conclusion: The findings show that RJE inhibits fibrosis production in HSCs and can be developed as a novel therapy for hepatic fibrosis. This is the first report showing the beneficial effects of $R$. javanica as an anti-fibrotic agent.
\end{abstract}

Keywords: Liver fibrosis, Rhus javanica, Cytotoxicity, Transforming growth factor-beta, Hepatic stellate cells

Tropical Journal of Pharmaceutical Research is indexed by Science Citation Index (SciSearch), Scopus, International Pharmaceutical Abstract, Chemical Abstracts, Embase, Index Copernicus, EBSCO, African Index Medicus, JournalSeek, Journal Citation Reports/Science Edition, Directory of Open Access Journals (DOAJ), African Journal Online, Bioline International, Open-J-Gate and Pharmacy Abstracts

\section{INTRODUCTION}

Liver fibrosis is a common consequence of chronic liver injury caused by a variety of mechanisms including oxidative damage, viral infection, and certain genetic diseases [1]. Fibrosis is defined by the excessive accumulation of the extracellular matrix (ECM) 
protein around inflamed or damaged tissue, which can lead to permanent scarring, organ malfunction including kidney disease, liver disease, idiopathic pulmonary fibrosis (IPF) and heart failure $[2,3]$.

Liver fibrosis received little attention until the 1980s, when hepatic stellate cells (HSCs), formerly known as lipocytes, Ito cells, or perisinusoidal cells, were identified as the main collagen-producing cells in the liver [4]. HSCs are presently regarded as one of the key cell types involved in the progression of liver fibrosis [5]. In normal liver, HSCs were described as being in a quiescent state. They represent $5-8 \%$ of the total number of liver cells [6].

The most intriguing aspect of the involvement of HSCs in liver fibrogenesis is that they undergo phenotypic changes often characterized as myofibroblastic activation [7]. The activation of HSCs to a proliferative, myofibroblastic phenotype plays a key role in hepatic fibrogenesis, since these cells are the principal cellular source of the excess collagen synthesis during hepatic fibrosis [8]. Activated HSCs secretes collagen scar for protection which underlies the recovery from liver diseases. However, if this is not recovered, this proceeds to liver fibrosis, liver cirrhosis and liver cancer [8]. Therefore, the prevention of liver fibrosis has great significance in ameliorating chronic liver diseases.

Use of herbal and natural products in the treatment of liver disorders has a long tradition and scientists have focused on certain natural products in the treatment of liver fibrosis [9]. In the search for novel promising agents from natural products for preventing and/or treating hepatic fibrosis, Rhus javanica Linn. extract from the family Anacardiaceae was evaluated. $R$. javanica, a deciduous arborescent plant, grows widely at the foot of mountains and ravines in Korea, China and other Asian countries. Traditionally, $R$. javanica fruit has been used as anti-diarrheal, anti-tussive, anti-coagulant and anti-perspirant. The leaves of $R$. javanica have been used as detoxicant, anti-venom for snake bites and are reported to possess hepatoprotective and antioxidant properties [10]. The parasitic cocoon known as "Gallunt" on $R$. javanica tree which contains gallotammin as major component, also has effects such as, antidiarrhea, hemostasis, anti-biotic, liver protection, detoxicant and anti-oxidant [10]. Reports also indicate that $R$. javanica has been widely used to treat cancer, dysentery, diarrhea, parasitic and bacterial infections in Korea, China, Japan and other Asian countries for centuries [11]. However, till date no evidence exists regarding the anti-fibrotic effects of $R$. javanica.

In the present investigation we evaluated the anti-fibrotic effects of $R$. javanica extract in an in vitro system using hepatic stellate cells (HSCs) and explored the intrinsic mechanisms involved.

\section{EXPERIMENTAL}

\section{Plant material}

The whole plant material of $R$. javanica was purchased from Plant Extract Bank, Daejeon, Korea. The material was authenticated by $\mathrm{Dr}$ Jong-Bo Kim, a taxonomist at Konkuk University, Korea, based on its microscopic and macroscopic characters. A voucher specimen (No: KKU-RJ13) was deposited at the herbarium of Konkuk University, Korea for future reference.

The dried plant material (50 g) was powdered and extracted with ethanol using Soxhlet's extraction technique for 3 days. The extract was then concentrated under reduced pressure at 45 ${ }^{\circ} \mathrm{C}$ and lyophilized (yield: $23.16 \%$, w/w). The lyophilized powder was dissolved in $10 \%$ dimethyl sulfoxide (DMSO; Sigma, St. Louis, $\mathrm{MO}$ ) and then filtered through a $0.22 \mu \mathrm{m}$ syringe filter and stored as stock until use.

\section{Isolation and culture of rat HSCs}

Specific pathogen-free male Wistar rats were purchased from Orient Bio (Seoul, Korea). The animal experimental protocol was approved by the Committee of Laboratory Animals according to institutional guidelines of Konkuk University, Korea. Animals were housed in conventional cages under control conditions of temperature $\left(23 \pm 3^{\circ} \mathrm{C}\right)$ and relative humidity $(50 \pm 20 \%)$, artificial light (12:12-h light: dark cycle, lights on at 0700) with free access to water and feed ad libitum prior to experiments. HSCs were isolated by in situ perfusion of livers from rats with collagenase and pronase, followed by differential centrifugation using Nycodenz (Histodenz ${ }^{\mathrm{TM}}$, Sigma, USA) density gradients as previously described [12]. HSCs were cultured in Dulbecco's modified essential medium (DMEM, GIBCO $尺$, USA) supplemented with $10 \%$ fetal bovine serum (FBS, GIBCO尺, USA) and $5 \%$ antibiotics in a humidified air containing $5 \% \mathrm{CO}_{2}$ at $37{ }^{\circ} \mathrm{C}$. Growth medium was changed on a daily basis for the first two days in culture, then every second day thereafter. Experiments described in this study were performed using cells between days 2 and 7 in culture. 


\section{Cell viability assay}

Cell viability assays were evaluated by the 3-(4, 5-dimethylthiazol-2yl)-2, 5-diphenyl-2Htetrazolium bromide (MTT) method. In a 96-well microplate, Chang cells $\left(7 \times 10^{3}\right.$ cells/well) were cultivated in DMEM medium supplemented as described previously [13]. Sample material was evaluated at various concentrations for $24 \mathrm{~h}$ at $37{ }^{\circ} \mathrm{C}$ in an atmosphere of $5 \% \quad \mathrm{CO}_{2}$ and $95 \%$ humidity. The cells were then incubated with MTT (5 mL; Sigma, USA) for $4 \mathrm{~h}$, and the reaction was interrupted by addition of dimethyl sulphoxide (DMSO, Sigma, USA). An ELISA reader was used to obtain the results at $540 \mathrm{~nm}$. The viabilities of the control cells were used as the control values at $100 \%$. All assays were performed in triplicate.

\section{Reverse transcription-polymerase chain reaction (RT-PCR)}

For RT-PCR, total RNA $(1 \mu \mathrm{g})$ was reversetranscribed using Moloney murine leukemia virus reverse transcriptase Super-Scriptll and an oligo $\mathrm{dT}$ primer (Invitrogen, USA) at $42{ }^{\circ} \mathrm{C}$ for $1 \mathrm{~h}$. cDNA $(1 \mu \mathrm{L})$ was amplified using specific primers as appropriate. Primer sets were as follows; COL1A2; forward: 5'-GCT GAG GGC AAC AGC AGA TTC-3', reverse:5'- G A T G T C C A G A G G T G C A A T G T C A A -3'; TGF- $\beta 1$; forward: 5'- C T G T C C A A A C T A A G G C T C G C 3', reverse: 5'- A C T G A A G C G A A A G C C C T G T A -3'; PDGF forward: 5'- C T G C C A C A G C A T G A T G A G A T T G A T -3', reverse: 5'- G C C A G G A T G G C T G A G A T C A C C A C -3'; a-SMA forward: 5'- G G T G C T G T C T C T C T A T G C C T C T G G A -3', reverse: 5'- C C C A T C A G G C A A C T C G A T A C T C T T C -3'; GAPDH; forward: 5'- G G C A C A G T C A A G C T G A G A T G -3', reverse: 5'- A T G G T G G T G A A G A G C C A G T A -3'. GAPDH was used to verify that equal amounts of RNA were used for RT-PCR amplification from different experimental conditions. Products were electrophoresed on a $1.5 \%$ agarose gel and visualized by staining with ethidium bromide.

\section{Western blot analysis}

Cells were lysed in protein extract buffer (30 $\mathrm{mM} / \mathrm{L}$ of Tris-hydrochloride at $\mathrm{pH} 8.0,10-\mathrm{mM} / \mathrm{L}$ of edetic acid, $1 \%$ Triton $\mathrm{X}-100,100 \mathrm{mM}$ of sodium chloride, and $2 \mathrm{mM}$ of phenylmethylsulfonyl fluoride), and then centrifuged for $1 \mathrm{~min}$ at $14,000 \mathrm{rpm}$. The proteins were separated by 10 $\%$ sodium dodecyl sulfate-polyacrylamide gel electrophoresis and transferred to a nitrocellulose membrane (Bio-Rad, USA). The membranes were then blocked for $1 \mathrm{~h}$ in Trisbuffered saline and Tween-20 buffer, consisting of $20 \mathrm{mM}$ of Tris-hydrochloride ( $\mathrm{pH} 7.6), 135 \mathrm{mM}$ of sodium chloride, $1 \%$ Tween- 20 , and $5 \%$ nonfat dry milk, and incubated at $4{ }^{\circ} \mathrm{C}$ for $8 \mathrm{~h}$ with monoclonal Smad antibody and polyclonal COL1A2 antibody. After incubation, the membrane was washed three times for $5 \mathrm{~min}$ in Tris-Buffered saline and Tween-20 buffer, and then treated with peroxidase-conjugated antirabbit IgG antibody (Vector Laboratories, Burlingame, CA) at a dilution of 1:1000 for $2 \mathrm{~h}$ at room temperature. The membrane was washed, and substrate was added from an enhanced chemiluminescence reagent kit (Du Pont, Boston, MA, USA) and detection is LAS-3000. As an internal control, $\beta$-actin expression was analyzed using $\beta$-actin antibody (Santa Cruz, USA).

\section{Statistical analysis}

Data are presented as mean \pm standard error of mean $(n=6)$. Differences between two groups were analyzed for statistical significance using Student's t-test. In all experiments, $p<0.05$ was considered to be statistically significant.

\section{RESULTS}

\section{Effect of RJE on Chang cell viability and morphology of HSCs}

As shown in Fig. 1, RJE treated at various concentrations $(100,300$ and $500 \mu \mathrm{g} / \mathrm{mL}$ ) did not show significant changes in the overall cell viability nor produced toxicity to Chang cells. However, treatment with $1000 \mu \mathrm{g} / \mathrm{mL}$ of RJS showed significant toxicity in Chang cells $(p<$ 0.001). Therefore, further experiments were carried using $500 \mu \mathrm{g} / \mathrm{ml}$ of RJE as it was safe concentration (Fig. 1).

\section{Effect of RJE on cell morphology in culture- activated HSCs}

Treatment with RJE $(500 \mu \mathrm{g} / \mathrm{mL})$ for $24 \mathrm{~h}$, on 8 day-cultured primary HSCs showed shrinking morphology (Fig. 3). Treatment with RJE decreased the number of viable HSCs, and the stretched fiber was decreased when compared to the untreated activated HSCs in $24 \mathrm{~h}$ at 500 $\mu \mathrm{g} / \mathrm{mL}$ (Fig 3B). 


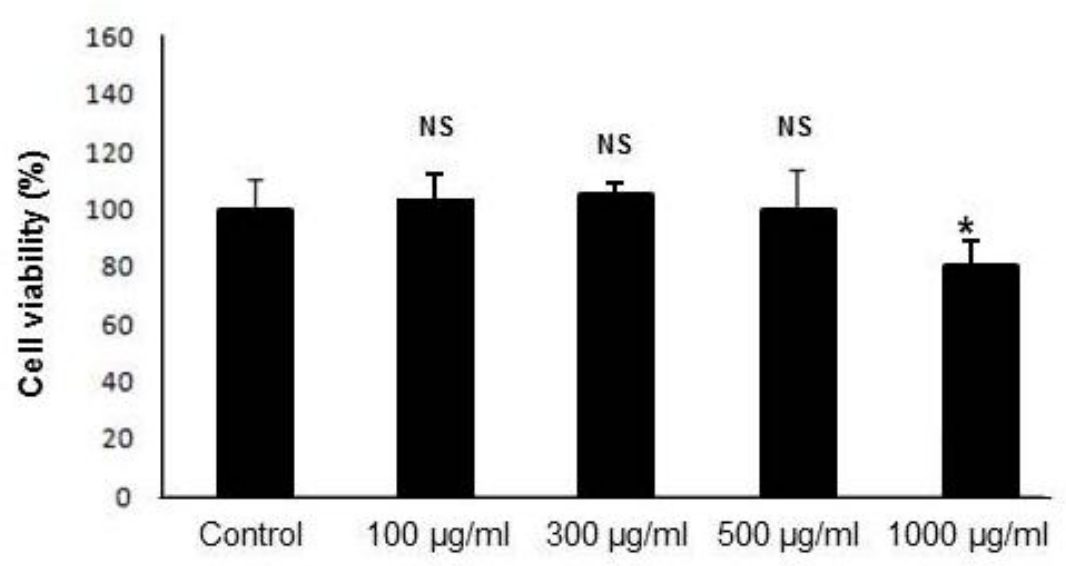

Fig 1: Cell viability of RJE in Chang liver cells. Data are presented as mean \pm SEM $(n=6)$. NS = not significant when compared with control groups; ${ }^{*} p<0.05$, when compared with control group; RJE = Rhus javanica extract.
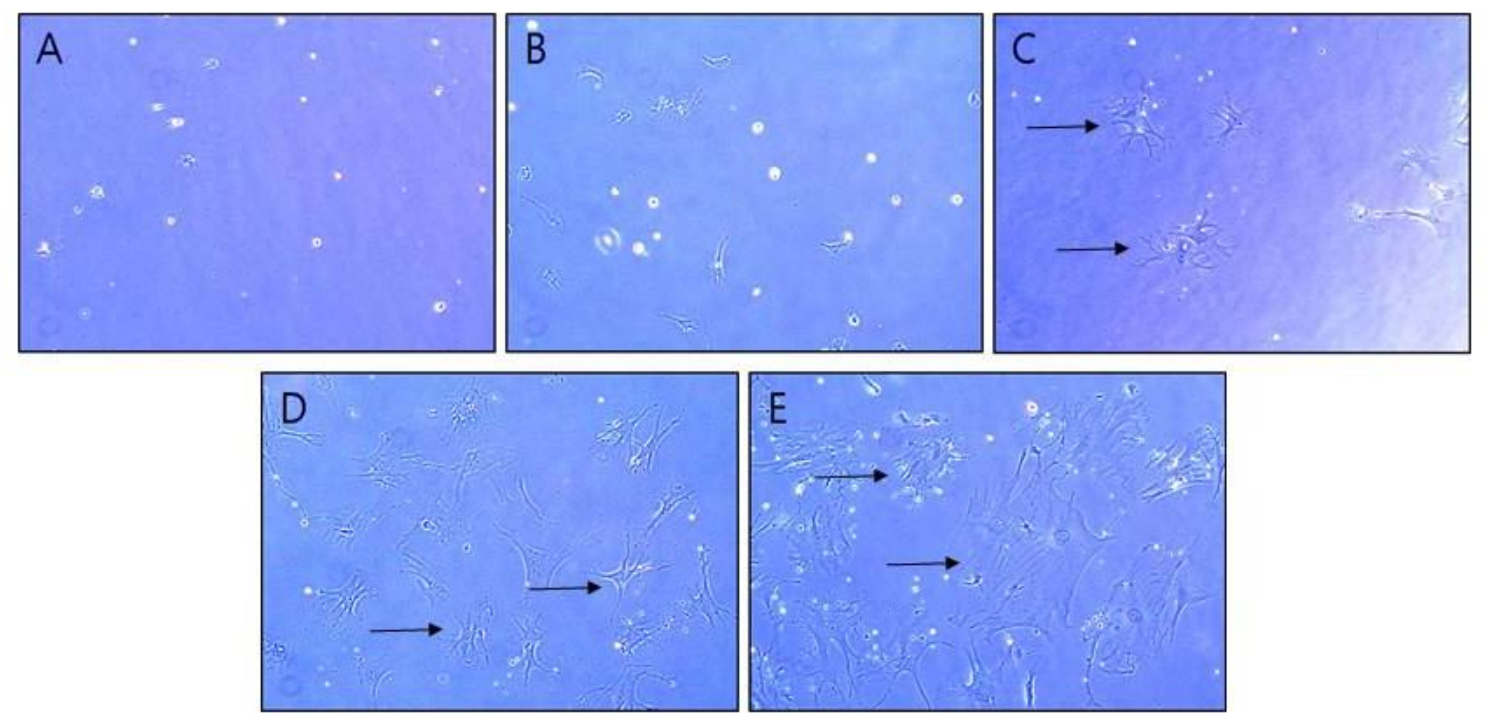

Fig 2: Morphology of isolated HSCs and activated HSCs. HSC was isolated for 1 day (A), 2 days (B), 4 days (C), 6 days $(D)$ and 8 days $(E)$. Microscope magnification $=x 100$. Arrows indicate HSCs. HSCs: Hepatic stellate cells

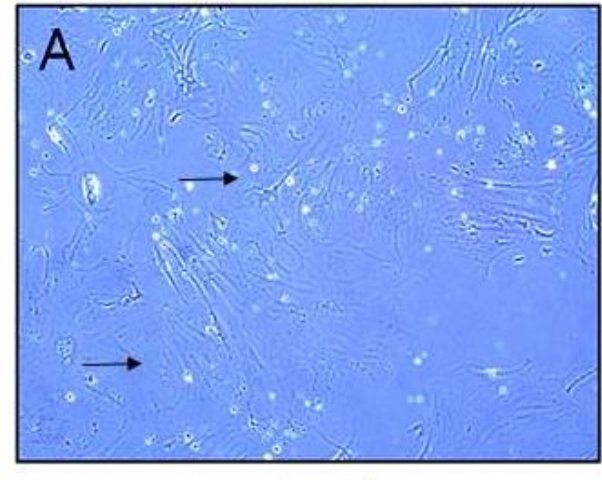

Control

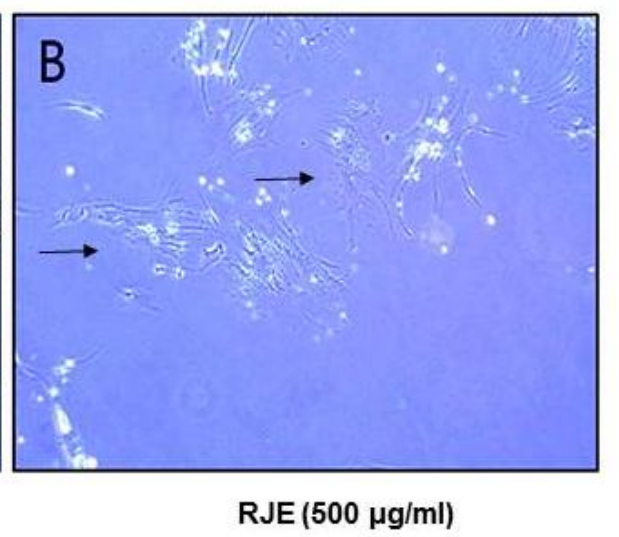

Fig 3: Effect of RJE on the activated HSCs morphologies. The cells were exposed to the indicated concentration $(500 \mu \mathrm{g} / \mathrm{mL})$ of RJE for $24 \mathrm{~h}$. Control cells were supplemented with same amount of vehicle medium. $A=$ activated HSCs and B = after $24 \mathrm{~h}$ treatment with RJE (B). Microscope magnification was $\times 100$. Arrows indicate HSCs; RJE = Rhus javanica extract; HSCs: Hepatic stellate cells 
Effect of RJE on COL1A2, $\alpha$-SMA, TGF- $\beta$ and PDGF mRNA expression in activated HSCs

As shown in Fig. 4A, in the presence of 500 $\mu \mathrm{g} / \mathrm{mL}$ of RJE the mRNA expressional levels of COL1A2, TGF- $\beta$ and $\alpha$-SMA were suppressed in activated HSC. However, RJE did not influence the PDGF expression in activated HSCs. Band intensities of related genes such as COL1A2, TGF- $\beta$ and $\alpha$-SMA revealed a significant decrease $(p<0.05)$ when treated with RJE at

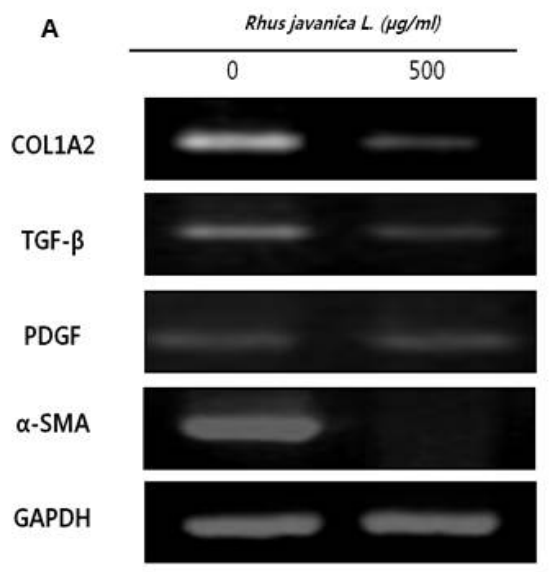

$500 \mu \mathrm{g} / \mathrm{mL}$ concentration suggesting that RJE inhibited the fibrosis production inhibited the fibrosis mediators in activated HSCs (Fig. 4B).

\section{Effect of RJE on the protein expressional levels of COL1A2 and Smad}

Treatment with RJE $(500 \mu \mathrm{g} / \mathrm{mL})$ suppressed the expressional levels of COL1A2 and Smad proteins (Fig 5).
B

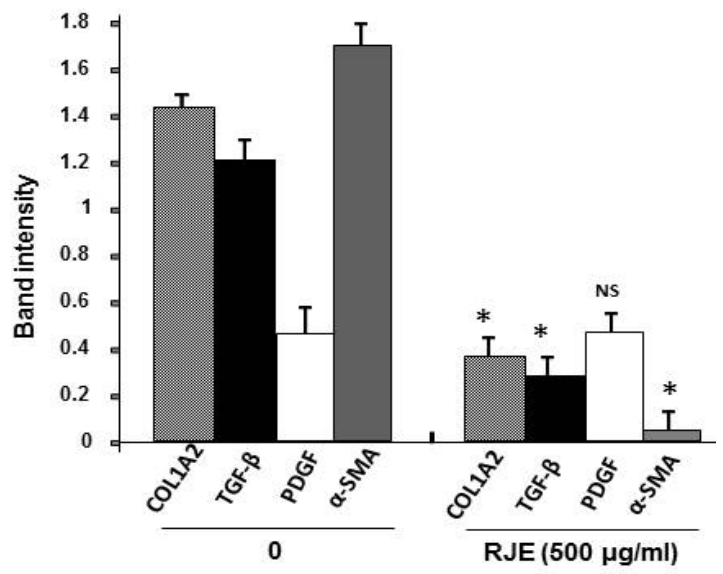

Fig 4: Effect of RJE on collagen expression of fibrosis-related genes and cytokines in activated HSCs. A = Cells were pretreated with RJE on activated HSCs for $24 \mathrm{~h}$. B = Band intensities of relative genes such as COL1A2, TGF- $\beta, \alpha-S M A$ and PDGF respectively. Data are presented as mean \pm SEM $(n=3)$; differences between two groups were analyzed for statistical significance using the Student's t-test; ${ }^{*} p<0.05$ when compared with COL1A2, TGF- $\beta$ and $\alpha-S M A$ genes respectively in untreated groups; NS = not significant when compared to PDGF gene expression in untreated group; RJE = Rhus javanica extract; HSCs: Hepatic stellate cells; COL1A2 = Collagen, type I, alpha 2; TGF- $\beta$ = Transforming growth factor-beta; PDGF = Platelet-derived growth factor; $\alpha-$ SMA = Alpha-smooth muscle actin and GAPDH = Glyceraldehyde 3-phosphate dehydrogenase
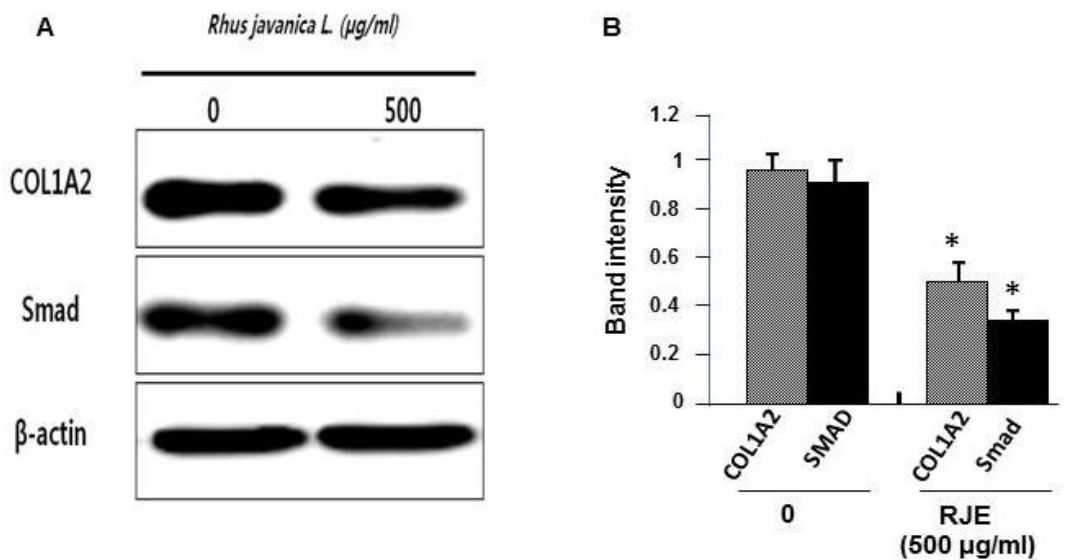

Fig 5: Effect of RJE on COL1A2 and Smad protein expressional levels in activated HSCs. $A=$ cells were pretreated with RJE to activated HSCs for $24 \mathrm{~h}$. B = band intensity of relative genes (COL1A2 and Smad, respectively). Data are presented as mean \pm SEM $(n=3)$. Differences between two groups were analyzed for statistical significance using the Student's t test; ${ }^{*} p<0.05$ when compared with COL1A2 and Smad, respectively, in untreated groups. RJE = Rhus javanica extract; HSCs: Hepatic stellate cells; COL1A2 = Collagen, type I, alpha 2 


\section{DISCUSSION}

Currently, antifibrotic therapeutic strategies include inhibition of HSC proliferation or stimulation of HSC apoptosis, down-regulation of collagen production or promotion of its degradation, administration of cytokines, and infusion of mesenchymal stem cells. Also, upregulation of collagen synthesis during activation is among the most striking molecular responses of HSCs to injury and is mediated by both transcriptional and post-transcriptional mechanisms. Transcriptional activation of type I collagen has been extensively characterized [14]. For that reason, HSCs plays a key role in hepatic fibrogenesis. Therefore HSC activation model can serve as a useful tool for screening liver fibrosis inhibition activities. Recently, several herbal and natural products including $R$. javanica have been used worldwide for the treatment of some liver diseases based on the plants' antioxidant defense mechanisms [10].

In the present investigation, RJE up to 500 $\mu \mathrm{g} / \mathrm{mL}$ did not exhibit any signs of cytotoxicity to HSCs. During the process of hepatic fibrosis HSCs become activated with characteristic cell proliferation and migration, production of collagen and other ECM molecules such as elastin, microfibrillar proteins, proteoglycans including hyaluronan, and noncollagenous glycoproteins, finally transforming into myofibroblasts. To obtain minimum contamination from other non-parenchymal cells, we used collagenase and pronase perfusion method for the isolation of hepatic stellate cells. This technique also allowed the simultaneous isolation of hepatocytes and HSCs.

It has been well known that HSCs are important anti-fibrotic targets and are considered as the main ECM producing cells in the injured liver [15]. Following chronic injury, HSCs activate or trans-differentiate into myofibroblast-like cells and accumulate increased ECM proteins. Hepatic fibrosis is associated with increased levels of TGF- $\beta$ which initially recruit inflammatory cells and fibroblasts into an area of injury and then stimulate these cells to produce cytokines and ECM, because TGF- $\beta$ not only enhances ECM synthesis but also inhibits ECM degradation [16,17]. HSCs up-regulate gene expression of ECM components, matrixdegrading enzymes and their respective inhibitors, resulting in matrix remodeling and accumulation at sites with abundant activated HSCs $[2,18]$.

PDGF and TGF- $\beta$ are the two best-characterized cytokines in stellate cell activation. A large number of studies identify PDGF as the main mediator for proliferation and TGF- $\beta$ as the most important cytokine stimulating fibrogenesis in stellate cells $[12,19]$. The fibrosis pathway includes a signaling crosstalk following TGF- $\beta$ binding to the receptor. Signal pathways potentially important here include the MAP kinase JNK. This would synergize with the Smad signaling pathway and decrease the activation of downstream TGF- $\beta$-dependent genes. Alternatively, NO could enhance the proteasomal degradation of Smad [20]. In the current study, down-regulation of fibrosis mediators by RJE was found to be due to the inhibition of TGF- $\beta 1$ pathway. Further, to verify the inhibitory mechanism of RJE on fibrosis we evaluated the phosphorylation of Smad two major TGF- $\beta 1$ pathway signaling proteins. The inhibitory action of RJE was mediated by prevention TGF- $\beta 1$ activation and by inhibition of Smad expression, which is accompanied by the blocking of TGF- $\beta 1$ pathway.

\section{CONCLUSION}

The findings indicate that RJE influenced the morphology of cultured/activated HSCs and inhibited fibrosis production enhanced by TGF- $\beta$ gene. RJE also inhibits expression of $\alpha$-SMA gene and collagen gene, and causes a marked blockage of Smad expression. Down-regulation of fibrosis mediators by RJE is due to inhibition of TGF- $\beta$ signaling pathway. This is the first report showing the beneficial effects of $R$. javanica as an anti-fibrotic agent, suggesting that it is a potential therapeutic agent for treating hepatic disorders.

\section{ACKNOWLEDGEMENT}

This work was supported by Konkuk University in 2014.

\section{REFERENCES}

1. Friedman SL. Liver fibrosis - from bench to bedside. J Hepatol 2003. 38 (Suppl. 1): S38-S53.

2. Bataller R, Brenner D. Liver fibrosis. J Clin Invest 2005; 115: 209-218.

3. Wynn TA. Integrating mechanisms of pulmonary fibrosis. J Exp Med 2011; 208: 1339-1350.

4. Friedman SL, Roll FJ, Boyles J, Bissell DM. Hepatic lipocytes: the principal collagen-producing cells of normal rat liver. Proc Natl Acad Sci U S A 1985; 82: 8681-8685.

5. Albanis E, Friedman SL. Hepatic fibrosis. Pathogenesis and principles of therpy. Clin Liver Dis 2001; 5: 315334.

Trop J Pharm Res, August 2015; 14(8): 1418 
6. Geerts A. History, heterogeneity, developmental biology, and functions of quiescent hepatic stellate cells. Semin Liver Dis 2001; 21: 311-335.

7. Miyahara $T$, Schrum L, Rippe $R$, Xiong $S$, Yee $H F$, Motomura $K$, Anania FA, Willson TM Tsukamoto $H$. Peroxisome proliferator-activated receptors and hepatic stellate cell activation. J Biol Chem 2000; 275: 35715-35722.

8. Pinzani M, Gesualdo L, Sabbah GM Abboud HE. Effects of platelet-derived growth factor and other polypeptide mitogens on DNA synthesis and growth of cultured rat liver fat-storing cells. J Clin Invest 1989; 84: 1786-1793.

9. Schuppan D, Jia JD, Brinkhaus B, Hahn EG. Herbal products for liver diseases: a therapeutic challenge for the new millennium. Hepatology 1999; 30: 10991104.

10. Ahn DK. Illustrated Book of Korean Medicinal Herbs. Kyo-Hak Publisher, Seoul, 1998.

11. You YO, Choi NY, Kang SY, Kim KJ. Antibacterial Activity of Rhus javanica against Methicillin-Resistant Staphylococcus aureus. Evid Based Complement Alternat Med 2013: 549207

12. Erawan $B K$, Jens $H$, Doris $S$, Jens $T$, Axel MG, Ralf $W$. Dominant-negative soluble PDGF- $\beta$ receptor inhibits hepatic stellate cell activation and attenuates liver fibrosis. Lab. Investig 2004; 84: 766- 777.

13. Twentyman PR, Luscombe M. A study of some variables in a tetrazolium dye (MTT) based assay for cell growth and chemosensitivity. Br J Cancer 1987; 56: 279-285.
14. Li JT, Liao ZX, Ping J, Xu D, Wang H. Molecular mechanism of hepatic stellate cell activation and antifibrotic therapeutic strategies. J Gastroenterol 2008; 43: 419-428.

15. Gabele E, Brenner DA, Rippe RA. Liver fibrosis: signals leading to the amplification of the fibrogenic hepatic stellate cell. Front Biosci 2003; 8: d69-77.

16. Baricos WH, Cortez SL, Deboisblanc M, Xin S. Transforming growth factor-beta is a potent inhibitor of extracellular matrix degradation by cultured human mesangial cells. J Am Soc Nephrol 1999; 10: 790795.

17. Tomooka S, Border WA, Marshall BC, Noble NA. Glomerular matrix accumulation is linked to inhibition of the plasmin protease system. Kidney Int 1992; 42, 1462-1469.

18. Knittel T, Kobold D, Dudas J, Saile B, Ramadori G. Role of the Ets-1 transcription factor during activation of rat hepatic stellate cells in culture. Am J Pathol 1999; 155: 1841-1848.

19. George J, Roulot D, Koteliansky VE, Bissell DM. In vivo inhibition of rat stellate cell activation by soluble transforming growth factor beta type II receptor: a potential new therapy for hepatic fibrosis. Proc Natl Acad Sci U S A 1999; 96: 12719-12724.

20. Dooley S, Streckert M, Delvoux B, Gressner AM. Expression of Smads during in vitro transdifferentiation of hepatic stellate cells to myofibroblasts. Biochem Biophys Res Commun 2001; 283: 554-562. 